\title{
PSMA-Ligand PET for Early Castration-Resistant Prostate Cancer: A Retrospective Single-Center Study
}

\author{
Manuel Weber*1, Claudia Kurek*1, Francesco Barbato ${ }^{1}$, Matthias Eiber ${ }^{2}$, Tobias Maurer ${ }^{3}$, Michael Nader ${ }^{1}$, \\ Boris Hadaschik $^{4}$, Viktor Grünwald ${ }^{5}$, Ken Herrmann ${ }^{1}$, Axel Wetter ${ }^{6}$, and Wolfgang P. Fendler ${ }^{1}$ \\ ${ }^{I}$ Department of Nuclear Medicine, University of Duisburg-Essen, and German Cancer Consortium-University Hospital Essen, Essen, \\ Germany; ${ }^{2}$ Department of Nuclear Medicine, Technical University Munich, Munich, Germany; ${ }^{3}$ Department of Urology and Martini- \\ Klinik, University of Hamburg-Eppendorf, Hamburg, Germany; ${ }^{4}$ Department of Urology, University of Duisburg-Essen, and German \\ Cancer Consortium-University Hospital Essen, Essen, Germany; ${ }^{5}$ Interdisciplinary Genitourinary Oncology, West German Cancer \\ Center, University Hospital Essen, Essen, Germany; and ${ }^{6}$ Institute of Diagnostic and Interventional Radiology and Neuroradiology, \\ University of Duisburg-Essen, and German Cancer Consortium-University Hospital Essen, Essen, Germany
}

\begin{abstract}
The low detection rate of conventional imaging and unspecific fluctuations in prostate-specific antigen can hamper early diagnosis of castration-resistant prostate cancer (CRPC). We thus assessed the value of prostate-specific membrane antigen (PSMA) PET/CT in the detection of early CRPC (prostate-specific antigen $\leq 3 \mathrm{ng} / \mathrm{mL}$ ). Methods: We identified 55 patients with early CRPC from our institutional database. PSMA PET/CT and its CT component were interpreted independently by 3 masked readers. The primary endpoint was the per-patient detection rate; secondary endpoints were interobserver agreement and predictors of PET positivity. Results: PSMA PET/CT was positive in 41 of $55(75 \%)$ patients. Sixteen of 55 (29\%) patients had local disease only, and 25 of 55 (45\%) had M1 disease. Overall, PSMA PET/CT interobserver agreement was substantial by Landis and Koch criteria (Fleiss $\mathrm{K}=0.77$ ). Conclusion: PSMA PET/CT localized prostate cancer lesions in $75 \%$ of patients and M1 disease in $45 \%$. Detection of early CRPC facilitates disease-delaying therapies for local or oligometastatic disease. PSMA PET/CT is of value in early CRPC and should be included in the CRPC entry criteria of the European Association of Urology and Prostate Cancer Working Group 3.
\end{abstract}

Key Words: CRPC; PCWG; PET; prostate cancer; PSMA

J Nucl Med 2021; 62:88-91

DOI: 10.2967/jnumed.120.245456

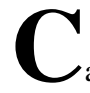

astration-resistant prostate cancer (CRPC) is characterized by biochemical or radiographic disease progression despite effective androgen deprivation therapy (1). Biochemical progression is defined as 3 consecutive prostate-specific antigen (PSA) rises, each at least $1 \mathrm{wk}$ apart, with two $50 \%$ increases over the nadir. However, pharmacologic androgen axis treatment was demonstrated to result in potentially disconnected effects on PSA expression and tumor growth (2-4). This finding is of importance in

Received Mar. 18, 2020; revision accepted Apr. 30, 2020.

For correspondence or reprints contact: Wolfgang Peter Fendler, University of Duisburg-Essen and German Cancer Consortium-University Hospital Essen, Hufelandstraße 55, 45147 Essen, Germany.

E-mail: wolfgang.fendler@uk-essen.de

${ }^{\star}$ Contributed equally to this work.

Published online May 22, 2020.

COPYRIGHT @ 2021 by the Society of Nuclear Medicine and Molecular Imaging. patients with a low but rising level of PSA as the only evidence of disease progression (5). Therefore, the diagnosis of CRPC requires repeat measurements and a bottom PSA threshold of $2.0 \mathrm{ng} / \mathrm{mL}$, according to the European Association of Urology (EAU), or $1.0 \mathrm{ng} / \mathrm{mL}$, according to Prostate Cancer Working Group 3 (PCWG3) (1,5). In addition, the assessment of radiographic progression is hampered considerably by low detection rates for a PSA level of $3 \mathrm{ng} / \mathrm{mL}$ or less. In recent years, systemic treatment in CRPC patients who are nonmetastatic by conventional imaging (nmCRPC) showed improvements in metastasisfree survival for apalutamide, darolutamide, and enzalutamide. In a prior study by our group, prostate-specific membrane antigen (PSMA) PET uncovered disease burden in almost all nmCRPC patients, demonstrating distant disease in 55\% and locoregional disease in only $44 \%$ of nmCRPC patients (6). Hence, in the era of precision medicine, the unprecedented accuracy of PSMA PET could lead the way toward a more personalized treatment strategy. We hypothesize that PSMA PET will improve stratification of nmCRPC candidates for local or systemic treatment even before PCWG3/EAU thresholds are met $(\mathrm{PSA} \leq 3 \mathrm{ng} / \mathrm{mL})$.

\section{MATERIALS AND METHODS}

Through screening of 1,965 prostate cancer patients in our institutional database, we identified 55 patients with histopathologically proven adenocarcinoma of the prostate who had undergone prostatectomy or primary radiotherapy, had a rising PSA level during continuous androgen deprivation therapy, and had a PSA level of less than $3 \mathrm{ng} / \mathrm{mL}$ at the time of PSMA PET/CT. Patients were stratified according to PSA level at imaging into 3 groups: less than $1.0 \mathrm{ng} / \mathrm{mL}$ (pre-PCWG group), $1.0 \mathrm{ng} / \mathrm{mL}$ to less than $2.0 \mathrm{ng} / \mathrm{mL}$ (early PCWG group), and $2.0 \mathrm{ng} / \mathrm{mL}$ to no more than $3.0 \mathrm{ng} / \mathrm{mL}$ (early EAU group). Nine patients were reported previously (6).

PSMA PET/CT was performed on a Siemens Biograph mCT device after the administration of a median of $110 \mathrm{MBq}$ (interquartile range, $35 \mathrm{MBq})$ of ${ }^{68} \mathrm{Ga}$-PSMA11 with a median uptake time of $70 \mathrm{~min}$ (interquartile range, $31 \mathrm{~min}$ ).

The PET/CT scan and the CT component were anonymized separately and interpreted visually by 3 independent masked readers in random order with at least $2 \mathrm{wk}$ between the PET/CT and the CT reading sessions. Lesion number, lesion size, and SUVs were assessed separately for 4 regions (prostate bed, pelvic lymph nodes, soft tissue including extrapelvic lymph nodes, and bones) and 21 subregions, as 
TABLE 1

Disease Extent Stratified by PSA at Time of Imaging According to PROMISE $(n=55)(17)$

\begin{tabular}{|c|c|c|c|c|}
\hline $\begin{array}{l}\text { PSMA PET/CT } \\
\text { findings }\end{array}$ & Total $(n=55)$ & $\begin{array}{c}\text { Pre-PCWG3 } \\
\text { PSA: }<1.0 \mathrm{ng} / \mathrm{mL} \\
(n=21)\end{array}$ & $\begin{array}{c}\text { Early PCWG3 } \\
\text { PSA: } 1.0-<2.0 \mathrm{ng} / \mathrm{mL} \\
(n=11)\end{array}$ & $\begin{array}{c}\text { Early EAU } \\
\text { PSA: } 2.0-\leq 3.0 \mathrm{ng} / \mathrm{mL} \\
(n=23)\end{array}$ \\
\hline Negative & $14(25)$ & $10(48)$ & $0(0)$ & $4(17)$ \\
\hline Tr/N1 only & $16(29)$ & $6(29)$ & $3(27)$ & $7(30)$ \\
\hline Local recurrence $(\mathrm{Tr})$ & $9(16)$ & $3(14)$ & $1(9)$ & $5(22)$ \\
\hline Pelvic lymph nodes (N1) & $9(16)$ & $3(14)$ & $2(18)$ & $4(17)$ \\
\hline Any $\mathrm{M} 1$ & $25(45)$ & $5(24)$ & $8(73)$ & $12(52)$ \\
\hline Extrapelvic lymph nodes (M1a) & $15(27)$ & $3(14)$ & $3(27)$ & $9(39)$ \\
\hline Bone (M1b) & $13(24)$ & $3(14)$ & $4(36)$ & $6(26)$ \\
\hline Soft tissue/visceral (M1c) & $2(4)$ & $0(0)$ & $2(18)$ & $0(0)$ \\
\hline $\mathrm{N} / \mathrm{M}$ disease extent & $34(62)$ & $8(38)$ & $0(0)$ & $16(70)$ \\
\hline Unifocal (1) & $6(18)$ & $2(25)$ & $1(10)$ & $3(19)$ \\
\hline Oligometastatic (2-5) & $17(50)$ & $4(50)$ & $9(90)$ & $4(25)$ \\
\hline Multiple or disseminated $(\geq 6)$ & $11(32)$ & $2(25)$ & $0(0)$ & $9(56)$ \\
\hline
\end{tabular}

Data are number of patients, followed by percentage in parentheses.

published previously (7). Statistical consensus was positive when at least 2 of 3 readers rated a region positive.

The primary endpoint was PSMA PET/CT versus CT lesion detection rate on a per-patient basis. Secondary endpoints were reproducibility, lesion detection stratified by PSA, and predictors for PET positivity or PET M1 disease.

Interobserver agreement was determined by Fleiss $\kappa$ and interpreted by the criteria of Landis and Koch (6). Odds ratio and corresponding 95\% confidence interval for PET positivity were assessed for different variables using multivariate analyses. Statistical analysis was performed with R, version 3.5.1, and SPSS software, version 24.0.

\section{RESULTS}

Median PSA at the time of PET was $1.5 \mathrm{ng} / \mathrm{mL}$; median patient age was $70 \mathrm{y}$. Twenty-seven of 44 patients $(61 \%)$ had a PSA doubling time of no more than $6 \mathrm{mo}$. Gleason score was at least 8 in 28 of 47 patients $(60 \%)$. Forty-four of 55 patients $(80 \%)$ had undergone primary prostatectomy, and 11 patients had undergone primary radiotherapy (20\%) (Supplemental Table 1; supplemental materials are available at http://jnm.snmjournals.org).

PSMA PET/CT detected prostate cancer lesions in 41 of 55 (75\%) patients, and CT alone detected prostate cancer lesions in 18 of $55(33 \%)$ patients. All CT lesions were also seen on PET/ CT. The per-patient detection rate for PSMA PET/CT stratified by PSA is shown in Table 1. In summary, 29\% (16/55) of patients had locoregional disease only, and 45\% (25/55) had M1 disease. Twentythree of $34(68 \%)$ patients with N/M findings had uni- or oligometastatic (2-5 lesions) disease. Interobserver agreement for PSMA PET/ CT was superior to CT (overall: $\kappa=0.77$ vs. 0.29 ; local tumor recurrence: $\kappa=0.75$ vs. 0.14 ; $\mathrm{N} 1$ disease: $\kappa=0.79$ vs. 0.53 ; M1a/c disease: $\kappa=0.91$ vs. 0.14 ; M1b disease: $\kappa=0.80$ vs. 0.47 ).

\section{DISCUSSION}

In line with prior publications, PSMA PET/CT detected lesions in $75 \%$ of patients with early CRPC even below PCWG3/EAU

\section{TABLE 2}

Regression Analysis of Clinical Parameters and Their Respective Risk for PSMA PET/CT Detection $(n=55)$

\begin{tabular}{|c|c|c|c|c|c|}
\hline Variable & $n$ & Odds ratio for PET-positive & $P$ & Odds ratio for PET M1 & $P$ \\
\hline Age $\geq 65$ & 40 of $55(73)$ & $1.3(0.3-4.9)$ & 0.75 & $0.9(0.3-3.1)$ & 0.91 \\
\hline Gleason score $\geq 8$ & 28 of $47(60)$ & $1.3(0.2-5.1)$ & 0.70 & $1.2(0.4-3.9)$ & 0.77 \\
\hline $\mathrm{PSA} \geq 1.5 \mathrm{ng} / \mathrm{mL}$ & 28 of $55(51)$ & $3.0(0.8-11.3)$ & 0.11 & $2.0(0.7-5.8)$ & 0.22 \\
\hline PSA doubling time $\leq 6$ mo & 27 of $44(61)$ & $0.8(0.2-3.5)$ & 0.82 & $1.3(0.4-4.2)$ & 0.70 \\
\hline Locoregional disease $\mathrm{pT} 3 / \mathrm{pT} 4^{\star}$ & 20 of $31(65)$ & $1.5(0.2-9.4)$ & 0.60 & $1.2(0.3-5.2)$ & 0.81 \\
\hline Locoregional disease $\mathrm{pN} 1^{\dagger}$ & 12 of $30(40)$ & $1.4(0.2-9.4)$ & 0.71 & $0.6(0.1-2.5)$ & 0.46 \\
\hline Primary radiation therapy & 11 of $55(20)$ & $0.5(0.1-2.1)$ & 0.36 & $0.1(0.0-0.7)$ & $0.02^{*}$ \\
\hline
\end{tabular}

${ }^{*}$ Analyzed for all patients after prostatectomy with known pT stage.

${ }^{\dagger}$ Analyzed for all patients after lymphadenectomy with known pN stage.

Data in parentheses are percentage or $95 \%$ confidence interval. 


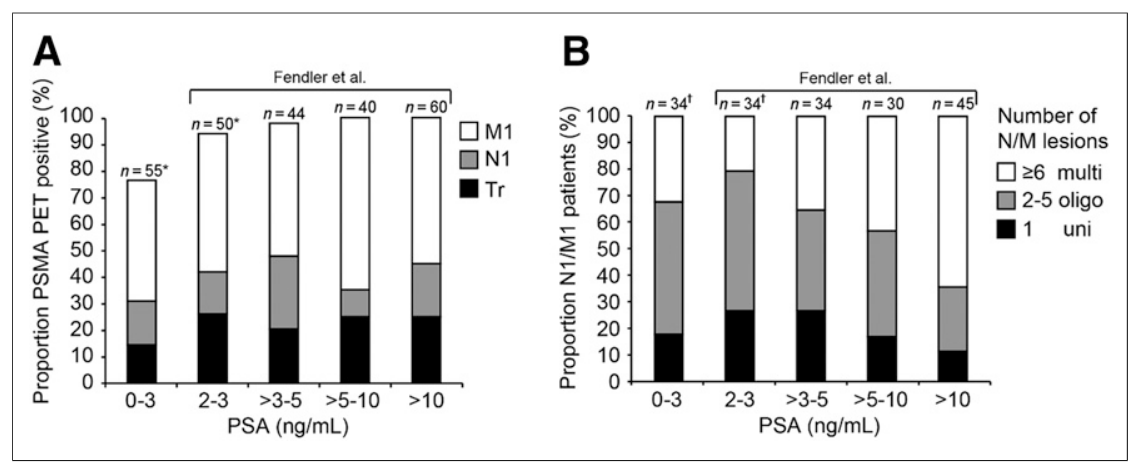

FIGURE 1. Proportion of local (Tr), nodal (N1), or distant (M1) disease (A) and N/M disease extent (B) stratified by PSA range for presented patients, including previous data by Fendler et al. (6). Six patients in study of Fendler et al. were excluded because of $P S A \geq 8$ wk before PET. multi $=$ multiple or disseminated; oligo $=$ oligometastatic; uni $=$ unifocal. ${ }^{*} n=9$ overlap. ${ }^{\dagger} n=5$ overlap.

thresholds and reliably distinguished local from distant disease, whereas $\mathrm{CT}$ demonstrated a low detection rate and slight reproducibility (8). Of note, PSMA PET resulted in stage migration to PET M1 disease in $45 \%$ of patients, potentially affecting management. Of the assessed risk factors, only primary radiation therapy was significantly associated with a lower rate of PET M1 disease $(P=$ 0.02 or 0.1 ), a finding that may be biased by an expected higher PSA nadir in this group; no other risk factor predicted PET positivity, indicating that PSMA PET/CT has additional value (Table 2).

In our prior study, PSMA PET localized extrapelvic disease in about half of nmCRPC patients with biochemical or histopathologic risk features (6). A joint post hoc analysis including Fendler et al. ( 6 ) and the presented patients demonstrated a higher proportion of metastatic disease and a lower proportion of uni- to oligometastatic findings with increasing PSA (Fig. 1). Thus, early diagnosis by PSMA PET/CT may provide additional value for disease-delaying metastasis-directed therapies. Although such treatments may postpone the start of other more toxic regimens, their impact on overall survival has not been demonstrated yet (9). Inversely, identification of distant disease on PSMA PET/CT may be an indicator of poor prognosis as shown previously by Emmett et al. in patients with biochemical recurrence (10). Consequently, the results of our study contribute to the growing body of evidence for a high prevalence of PET M1 disease in nmCRPC patients. The degree of upstaging by PSMA PET/CT depends on the extent of conventional imaging; however, a prior head-to-head comparison indicates that additional MRI and bone scans have a low impact on early detection of metastases (11-13). In light of recent clinical trials showing improved outcome in nmCRPC patients, such as SPARTAN (14) or ARAMIS (15), we assume that about half of subjects enrolled in these trials did indeed have metastatic disease detectable by PET. The clinical significance of PET M1 disease in CRPC patients, however, has yet to be determined.

PSMA PET/CT has become standard-of-care imaging for biochemical recurrence after prostatectomy or radiotherapy. We anticipate large patient groups with available PSMA PET/CT staging at baseline before a subsequent PSA rise. Initial work to follow up PSMA PET/CT progression may create new criteria for CRPC. Frameworks to assess PSMA PET/CT disease progression have been proposed (16). Given the limitations of PSA, such as unspecific fluctuations in the low detection range, PSMA PET/CT may serve as a complementary or even independent biomarker of early CRPC tumor load (2).
Limitations of our study include its retrospective single-center design, small sample size, lack of serum testosterone levels at the time of PET, and missing correlational bone scans or MRI scans in some patients.

\section{CONCLUSION}

PSMA PET/CT detects prostate cancer in most patients with early CRPC even below the valid EAU/PCWG3 PSA thresholds are reached. Early staging is associated with a higher rate of targetable local or uni- to oligometastatic disease, which may provide value for metastasis-directed therapy. Now, most patients with biochemical recurrence will undergo baseline PSMA PET/ $\mathrm{CT}$, and any PET-based disease progression under effective androgen deprivation may serve as new entry criteria for CRPC. These aspects need attention in future clinical trials on CRPC imaging and targeted therapy.

\section{DISCLOSURE}

Manuel Weber is on the speakers' bureau for Boston Scientific. Boris A. Hadaschik has an advisory role with ABX, Bayer, Lightpoint Medical, Inc., Janssen R\&D, Bristol-Myers-Squibb, and Astellas; research funding from Profound Medical, German Cancer Aid, German Research Foundation, Janssen R\&D, BristolMyers-Squibb, and Astellas; and travel support from AstraZeneca, Janssen R\&D, and Astellas. Wolfgang P. Fendler is a consultant for Ipsen, Endocyte, and Boston Scientific; received personal fees from RadioMedix outside the submitted work; and received financial support from the German Research Foundation (Deutsche Forschungsgemeinschaft, DFG, grant FE1573/3-1/659216), Mercator Research Center Ruhr (MERCUR, An-2019-0001), IFORES (D/107-81260, D/107-30240), Doktor Robert Pfleger-Stiftung, and Wiedenfeld-Stiftung/Stiftung Krebsforschung Duisburg. Tobias Maurer has an advisory role with BlueEarth Diagnostics and ROTOP. Matthias Eiber has an advisory role with Blue Earth Diagnostics and a patent application for rhPSMA. No other potential conflict of interest relevant to this article was reported.

\section{ACKNOWLEDGMENT}

This work is part of the MD thesis of Claudia Kurek.

\section{KEY POINTS}

QUESTION: Can PSMA PET accurately localize prostate cancer in nmCRPC patients with beginning castration resistance?

PERTINENT FINDINGS: PSMA PET reveals prostate cancer in most patients and metastatic disease in almost half of the study cohort.

IMPLICATIONS FOR PATIENT CARE: PSMA PET has the potential to complement PSA and radiographic assessment in the detection of disease progression.

\section{REFERENCES}

1. Mottet N, Bellmunt J, Briers E, et al. EAU-ESTRO-ESUR-SIOG guidelines on prostate cancer. European Association of Neurology website. https://uroweb.org/ wp-content/uploads/09-Prostate-Cancer_2017_web.pdf. Published 2017. Accessed July $6,2020$. 
2. Bubley GJ, Carducci M, Dahut W, et al. Eligibility and response guidelines for phase II clinical trials in androgen-independent prostate cancer: recommendations from the Prostate-Specific Antigen Working Group. J Clin Oncol. 1999;17: 3461-3467.

3. Denmeade SR, Sokoll LJ, Dalrymple S, et al. Dissociation between androgen responsiveness for malignant growth vs. expression of prostate specific differentiation markers PSA, hK2, and PSMA in human prostate cancer models. Prostate. 2003;54:249-257.

4. Dixon SC, Knopf KB, Figg WD. The control of prostate-specific antigen expression and gene regulation by pharmacological agents. Pharmacol Rev. 2001;53: 73-91.

5. Scher HI, Morris MJ, Stadler WM, et al. Trial design and objectives for castrationresistant prostate cancer: updated recommendations from the Prostate Cancer Clinical Trials Working Group 3. J Clin Oncol. 2016;34:1402-1418.

6. Fendler WP, Weber M, Iravani A, et al. Prostate-specific membrane antigen ligand positron emission tomography in men with nonmetastatic castration-resistant prostate cancer. Clin Cancer Res. 2019;25:7448-7454.

7. Fendler WP, Calais J, Allen-Auerbach M, et al. ${ }^{68} \mathrm{Ga}$-PSMA-11 PET/CT interobserver agreement for prostate cancer assessments: an international multicenter prospective study. J Nucl Med. 2017;58:1617-1623.

8. Fendler WP, Calais J, Eiber M, et al. Assessment of ${ }^{68}$ Ga-PSMA-11 PET accuracy in localizing recurrent prostate cancer: a prospective single-arm clinical trial. JAMA Oncol. 2019;5:856-863.

9. Ost P, Reynders D, Decaestecker K, et al. Surveillance or metastasis-directed therapy for oligometastatic prostate cancer recurrence: a prospective, randomized, multicenter phase II trial. J Clin Oncol. 2018;36:446-453.
10. Emmett L, Tang R, Nandurkar R, et al. 3-year freedom from progression after 68Ga-PSMA PET/CT-triaged management in men with biochemical recurrence after radical prostatectomy: results of a prospective multicenter trial. $\mathrm{J} \mathrm{Nucl} \mathrm{Med}$. 2020;61:866-872.

11. Emmett L, Metser U, Bauman G, et al. Prospective, multisite, international comparison of $18 \mathrm{~F}$-fluoromethylcholine PET/CT, multiparametric MRI, and 68Ga-HBED-CC PSMA-11 PET/CT in men with high-risk features and biochemical failure after radical prostatectomy: clinical performance and patient outcomes. J Nucl Med. 2019;60:794-800.

12. Pyka T, Okamoto S, Dahlbender M, et al. Comparison of bone scintigraphy and ${ }^{68} \mathrm{Ga}$-PSMA PET for skeletal staging in prostate cancer. Eur J Nucl Med Mol Imaging. 2016;43:2114-2121.

13. Dyrberg E, Hendel HW, Huynh THV, et al. ${ }^{68} \mathrm{Ga}$-PSMA-PET/CT in comparison with ${ }^{18} \mathrm{~F}$-fluoride-PET/CT and whole-body MRI for the detection of bone metastases in patients with prostate cancer: a prospective diagnostic accuracy study. Eur Radiol. 2019;29:1221-1230.

14. Smith MR, Saad F, Chowdhury S, et al. Apalutamide treatment and metastasisfree survival in prostate cancer. N Engl J Med. 2018;378:1408-1418.

15. Fizazi K, Shore N, Tammela TL, et al. Darolutamide in nonmetastatic, castration-resistant prostate cancer. N Engl J Med. 2019;380:1235-1246.

16. Fanti S, Hadaschik B, Herrmann K. Proposal for systemic-therapy responseassessment criteria at the time of PSMA PET/CT imaging: the PSMA PET progression criteria. J Nucl Med. 2020;61:678-682.

17. Eiber M, Herrmann K, Calais J, et al. Prostate Cancer Molecular Imaging Standardized Evaluation (PROMISE): proposed miTNM classification for the interpretation of PSMA-ligand PET/CT. J Nucl Med. 2018;59:469-478. 